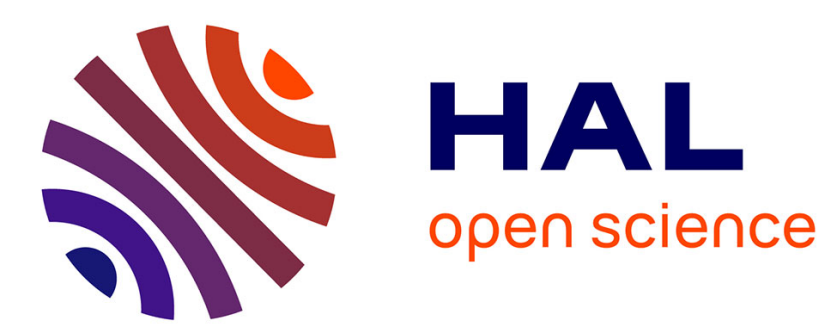

\title{
Etude expérimentale des écoulements darcéens à travers un lit de fibres rigides empilées aléatoirement : influence de la porosité
}

\author{
O. Rahli, L. Tadrist, Marc Miscevic, R. Santini
}

\section{- To cite this version:}

O. Rahli, L. Tadrist, Marc Miscevic, R. Santini. Etude expérimentale des écoulements darcéens à travers un lit de fibres rigides empilées aléatoirement : influence de la porosité. Journal de Physique II, 1995, 5 (11), pp.1739-1756. 10.1051/jp2:1995211 . jpa-00248266

HAL Id: jpa-00248266

https://hal.science/jpa-00248266

Submitted on 1 Jan 1995

HAL is a multi-disciplinary open access archive for the deposit and dissemination of scientific research documents, whether they are published or not. The documents may come from teaching and research institutions in France or abroad, or from public or private research centers.
L'archive ouverte pluridisciplinaire HAL, est destinée au dépôt et à la diffusion de documents scientifiques de niveau recherche, publiés ou non, émanant des établissements d'enseignement et de recherche français ou étrangers, des laboratoires publics ou privés. 
Classification

Phvsics Abstracts

$4755 \mathrm{Mh}-4715-\mathrm{x}$

\title{
Etude expérimentale des écoulements darcéens à travers un lit de fibres rigides empilées aléatoirement : influence de la porosité
}

\author{
O Rahli, L. Tadrist, M. Miscevic et R. Santini \\ Laboratoıre de l'Instıtut Unıversitaıre des Systèmes Thermıques Industrıels (IUSTI)( ${ }^{*}$ ), \\ Unıversité de Provence, Centre de St Jérôme, Case 162, Avenue Escadrille Normandıe Niemen, \\ 13397 Marselle Cedex 20, France
}

(Received 14 February 1995, revised 21 June 1995, accepted 24 July 1995)

\begin{abstract}
Résumé. - Des études expérımentales de l'écoulement d'un flùde à travers un mlleu poreux constıtué de fibres monodısperses empılées aléatoirement sont réalısées Les milıeux fibreux étudiés ont des porosités variant de 0,35 à 0,90 Ces porosités sont obtenues à l'aide de fibres dont le rapport d'aspect $L / d$ varie de 4 à 70 Les lors de perte de charge en fonction de la vitesse du fluide sont détermınées systématıquement pour chaque milıeu poreux Une analyse détallée est effectuée pour les farbles vitesses d'écoulement L'influence de la dırection de l'écoulement sur les loıs de perte de charge est étudiée. Celles-ci sont établıes survant deux directions perpendicularres Les résultats obtenus mettent en évidence un comportement du milieu fibreux globalement isotrope vis à vis de l'écoulement du flunde La perméabılité et le paramètre de Kozeny Carman $k_{\mathrm{k}}$ sont déduits des résultats expérımentaux Les variatıons de la perméabilité en fonction de la porosité suivent une lo1 de type exponentıel Le paramètre de Kozeny Carman $k_{\mathrm{k}}$ décroît en fonction de la porosité $\epsilon(L / d)$ et tend asymptotıquement vers une valeur proche de celle déduıte de la relation d'Ergun modıfiée. La décroissance importante, observée pour les petits rapports d'aspect, est sans doute due aux effets induits par les surfaces de base des fibres Ces effets deviennent néglıgeables pour les grands rapports d'aspect Les résultats en terme de perméabılité et de paramètre de Kozeny Carman sont comparés systématıquement avec les modèles proposés dans la littérature Généralement, ces modèles supposent des cylindres de grande longueur, dısposés en réseaux sımples L'écoulement est soıt parallèle. solt perpendıcularre à l'axe des cylındres Les lois de varıation du paramètre $k_{k}$, déduıtes des différents modèles, présentent des écarts importants avec les résultats expérimentaux Ces résultats semblent montrer qu'une disposition des fibres en réseaux simples ne permet pas une description convenable des écoulements à travers les emplements aléatorres de fibres
\end{abstract}

\begin{abstract}
Experımental studies have been carried out on fluld flow through porous media made up of randomly packed monodisperse fibers The fibers of fixed diameter have an aspect ratio $(L / d)$ varying between 4 and 70 given porosities of the porous media varying between 035 and 090 The relationships between friction losses and superficial velocity have been systematically determined for each porous medium A detalled analysis is carried out for low fluid velocities The influence of flow direction on pressure drop is studied along two perpendicular directions it is found that fibrous media behave globally in isotropic manner The permeability
\end{abstract}

$\left({ }^{*}\right)$ U M R 139 du CNRS

(C) Les Editıons de Physıque 1995 
and the Kozeny Carman parameter $k_{\mathrm{k}}$ are deduced from expermental results The variations of the permeability increase exponentially with the porosity The Kozeny Carman parameter $k_{\mathrm{k}}$ is a decreasing function of the porosity $\epsilon(L / d)$ and tends asymptotically to a value close to that deduced from a modified Ergun relation The important decrease, observed for small aspect ratios, is certainly an effect of the cut sections of fibers This effect becomes negligible for larger aspect ratios The results in terms of permeability and of Kozeny Carman parameter $k_{k}$ are systematically compared to those deduced from various theoretical models Generally, these models consider cylinders arranged in simple network, the flow being either parallel or perpendicular to the axis of cylinders The variation laws of the parameter $k_{\mathrm{k}}$, deduced from different models, present important discrepancies with our experimental results The theoretical models. established for regular arrays of fibers do not correctly describe the behavior of randomly packed fibers

\section{Introduction}

Les mileux poreux jouent un rôle important dans un certain nombre d'applications industrielles Parmı ces milıux, certains sont composés de fibres, ou de partıcules pouvant être assimilées à des fibres Citons par exemple les chaînes de polymères en solution, la laine de verre. les filtres, les matérıaux d'isolatıon, les céramıques rétıculées. Ces milieux présentent des porosités importantes Dans le cas de fibres monodisperses empilées aléatorrement. la porosité est fonction du rapport d'aspect des fibres $[1,2]$. Ces auteurs ont déterminé expérimentalement la loi de variation de la porosité en fonction du rapport d'aspect des fibres Celle-c1 varie de 0,35 à 0,90 pour des rapports d'aspect variant de 4 à 70 . contrairement au cas des empilements de particules sphériques où la porosité est comprise entre 0,36 et 0.40

Les travaux concernant les écoulements de fluides à travers des empilements aléatoires de fibres monodisperses sont, à notre connaissance, limités. La complexıté du milieu rend difficıle l'obtention d'une loi analytıque d'écoulement L'approche expérimentale demeure à l'heure actuelle la mieux adaptée pour traiter ce type de problème.

Jackson et James [3] ont effectué une revue bibliographique exhaustive tant des travaux expérımentaux que théoriques de détermination de la perméabilité

Les données expérımentales recueilles sont relatives à 25 milieux fibreux, avec des diamètres de fibres allant de $10 \AA$ à $10 \mathrm{~mm}$ Dans tous les cas, le rapport d'aspect des fibres est supposé suffisamment grand pour que seul le diamètre soit la dımension caractérıstıque de la partıcule.

Les modèles proposés considèrent diverses géométrıes d'empilement Les fibres sont rangées en réseaux parallèles ou perpendicularres à l'écoulement Dans le cas d'empllements aléatoures, les modèles sont déduıts de la combınaison des modèles précédents

Ces auteurs soulıgnent l'importance de l'alıgnement et de l'homogénéité de la distribution des fibres sur la perméabilité du milieu poreux

De nombreuses valeurs expérimentales de perméabilité pour différentes porosités sont proposées dans la littérature [3-5, .] Cependant aucune lo1 générale de variation ne peut être déduite du fait de la diversité des milieux utılisés En effet, ces valeurs sont très spécifiques à chaque expérimentation car les milieux utılisés ne sont pas des emplements de partıcules ayant une géométrie bien définie (filtre, laıne de verre, laine de roche..)

Dans le but de prédire la perméabllté d'un emplement aléatoire de fibres, Jackson et James [3] proposent un modèle basé sur un réseau cubique. Par additıon des pertes de charge des écoulements perpendıculaire et parallèle aux particules, et par évaluation des résıstances 
individuelles par la formule de Drummond et Tahır [6], ils en déduisent la relation .

$$
\frac{K}{d^{2}}=\frac{3}{5}\left(-\operatorname{Ln}(1-\epsilon)-0,931+O(\operatorname{Ln}(1-\epsilon))^{-1}\right)
$$

où $d$ est le diamètre des fibres et $\epsilon$ est la porosité du milıeu

L'écoulement perpendiculaıre ou parallèle à un réseau de cylindres est généralement étudié à partır d'une cellule élémentaıre modèle représentative du milieu. Dans l'étude de l'écoulement parallèle aux cylindres effectuée par Happel and Brenner [7], une solution est déterminée pour un écoulement unidirectionnel dans un canal annulaire délımité à l'intérieur par un cylindre solide (diamètre $d$ ), et à l'extérıeur (diamètre $d+\delta$ ) par une surface libre. Ce cylindre extérieur représente le domaine d'interaction du cylindre solide avec son environnement. Ces auteurs trouvent, pour la "constante" de Kozeny Carman $k_{\mathrm{k}}$ (vorr relation (9))

$$
k_{\mathrm{k}}=\frac{2 \epsilon^{3}}{(1-\epsilon)\left[2 \operatorname{Ln} \frac{1}{1-\epsilon}-3+4(1-\epsilon)-(1-\epsilon)^{2}\right]}
$$

Pour un écoulement perpendiculaıre aux cylindres, le paramètre $k_{\mathrm{k}}$ a pour expressıon :

$$
k_{k}=\frac{\frac{2 \epsilon^{3}}{(1-\epsilon)}}{\operatorname{Ln} \frac{1}{1-\epsilon}-\frac{1-(1-\epsilon)^{2}}{1+(1-\epsilon)^{2}}}
$$

Dullien [8] propose un modèle qui consıste en des capillaires contenant des segments de sectıons droites varıables Ces capillaires sont arrangés selon des combınaisons séries parallèles.

Dans le cas d'un arrangement aléatorre de cylindres, Happel et Brenner [7] proposent pour $k_{\mathrm{k}}$ une combinaison linéarre des paramètres de Kozeny Carman calculés pour des écoulements parallèles et perpendiculaires

Pour des écoulements de fluides à travers des lits fibreux, Kyan et al. [9], relèvent l'existence d'une grande perte de charge inattendue, cela en dépit de la porosité importante du lit. Ils attribuent cette élévation à plusieurs effets

- seule une fraction de l'espace libre telle qu'elle est calculée à partir de la densité volumique du lit est traversée par le fluide, le reste sera occupé par du fluide stagnant ;

- une certaine énergie est absorbée par déformatıon des fibres individuelles causant une perte de charge additionnelle.

Ces auteurs postulent que la perte de charge totale à travers le lit fibreux est la somme de la perte de charge due aux pertes visqueuses, à la force de trainée, et aux déformations par flexion des fibres Ils proposent une corrélation donnant le paramètre $k_{\mathrm{k}}$ en fonction de la poısité du milieu et du nombre de pores effectıfs $N_{\mathrm{e}}$ définı par la relation .

$$
N_{\mathrm{e}}=\sqrt{\frac{\pi}{(1-\epsilon) \sin a}}-2,5
$$

où $\alpha$ est l'angle d'inclınaison entre deux fibres adjacentes Les auteurs préconısent de prendre $\alpha$ égal à $30^{\circ}$. Le paramètre $k_{\mathrm{k}}$, a alors pour expression .

$$
k_{\mathrm{k}}=\frac{\left[62,3 N_{\mathrm{e}}^{2}(1-\epsilon)+107,4\right] \epsilon^{3}}{16 N_{\mathrm{e}}^{6}(1-\epsilon)^{4}}
$$


L'ensemble des travaux expérimentaux présentés dans la littérature considèrent des milieux fibreux de grandes porosités, où la géométrıe des fibres n'est pas bien définı. En règle générale, les auteurs consıdèrent que seul le diamètre est la dimension caractérıstıque des particules.

Dans la présente étude, nous déterminons les lois d'écoulement, la perméabilité, et le paramètre $k_{\mathrm{k}}$, d'un milieu poreux constıtué d'un empilement aléatorre de fibres rigıdes monodisperses. Pour l'ensemble de nos experiences, le diamètre des fibres a été maintenu constant et égal à $150 \mu \mathrm{m}$ Nous présentons une descriptıon détaillée du dispositif expérimental et du mode opératoire adopté. Les paragraphes sulvants sont consacrés à l'établissement des lois de perte de charge en fonction de la vitesse apparente du fluide. Dans un premier temps, nous présentons les analyses relatives à l'influence de la direction de l'écoulement par rapport à la direction de l'emplement Celle ci est définie comme étant la direction d'introduction des fibres pour réaliser l'empilement Nous étudions, ensuite, l'influence du rapport d'aspect " $r$ " des fibres et de la porosité sur la perméabilité Une lo1 d'évolution du paramètre $k_{\mathrm{k}}$ en fonction de ces deux grandeurs est proposée.

La dernıère partıe est consacrée à l'analyse et à l'interprétatıon des résultats expérimentaux Une comparaison est effectuée avec les résultats expérımentaux ou issus des modèles disponıbles dans la littérature pour des milieux poreux simnlaires à ceux de la présente étnde.

\section{Dispositif expérimental}

Nous avons mis en oeuvre un dispositif automatıque permettant de fabriquer des fibres monodisperses. Pour cela, nous disposons de bobınes de fil métallique (cuivre, bronze) de $150 \mu \mathrm{m}$ de diamètre Une commande électronique règle l'avance d'un moteur pas à pas et la fermeture d'une pince coupante. Ceci permet d'entraîner le fil et de le découper en fibres à la longueur choisie (Fıg. 1a) La machine mise en oeuvre peut découper plusieurs fils simultanément. Le tranchant de la pince ne produit pas une section droite de la fibre Néanmoins, on peut admettre que la dimension du biseau est négligeable devant la longueur de la fibre (Fig. 1b) Dans le cas des fibres de cuivre, pour des rapports d'aspect supéreurs à 30. celles-ci peuvent se courber facilement et par conséquent des déformations importantes peuvent être occasionnées lors de l'emplement Par contre le fil de bronze est plus riglde et résiste mieux à la flexion pour des rapports d'aspect allant jusqu'à 80 Il est à noter que, pour des rapports d'aspects supérieurs à 100, la déformatıon est importante quelle que soit la nature des fibres utılisées.

Les fibres ainsı fabriquées sont empilées aléatoirement dans une cellule de mesure en les tassant progressivement et en fassant vibrer la cellule La porosité est ensuite déterminée par pesée des fibres contenues dans la cellule. Cette opératıon est réalısée pour chaque rapport d'aspect des fibres [1].

Afin de déterminer expérımentalement les lois de perte de charge à travers les milieux fibreux, nous avons réalısé un dispositif expérimental représenté sur la figure 2a.

La cellule (C) contenant les fibres est placée entre deux réservoirs ( $A, B)$ identıques et interconnectés. Deux surverses permettent de mantenir les niveaux d'eau constants dans ces deux réservoirs L'excès de liquide est récupéré dans le réservoir $(E)$. La pompe $(P)$ remonte en permanence l'eau du réservoir $(E)$ dans le réservoir $(A)$

Le mode opératoire consiste à imposer une dufférence de hauteur fixe entre les niveaux de liquide dans les deux réservoirs $(A)$ et $(B)$. L'eau est ainsı mise en circulation à travers le milieux poreux. Au début de chaque expérience, lors du remplissage en eau de la boucle, de l'air résiduel reste prégé dans le milieu poreux Son évacuation est réalisée par circulation du liquide pendant plusieurs heures Les variations de la perte de charge en fonction de la vitesse apparente du fluide sont déterminées systématıquement en régime permanent La hauteur plézométrique $\Delta H$ est mesurée entre l'entrée et la sortie du milieu fibreux. Une éprouvette 


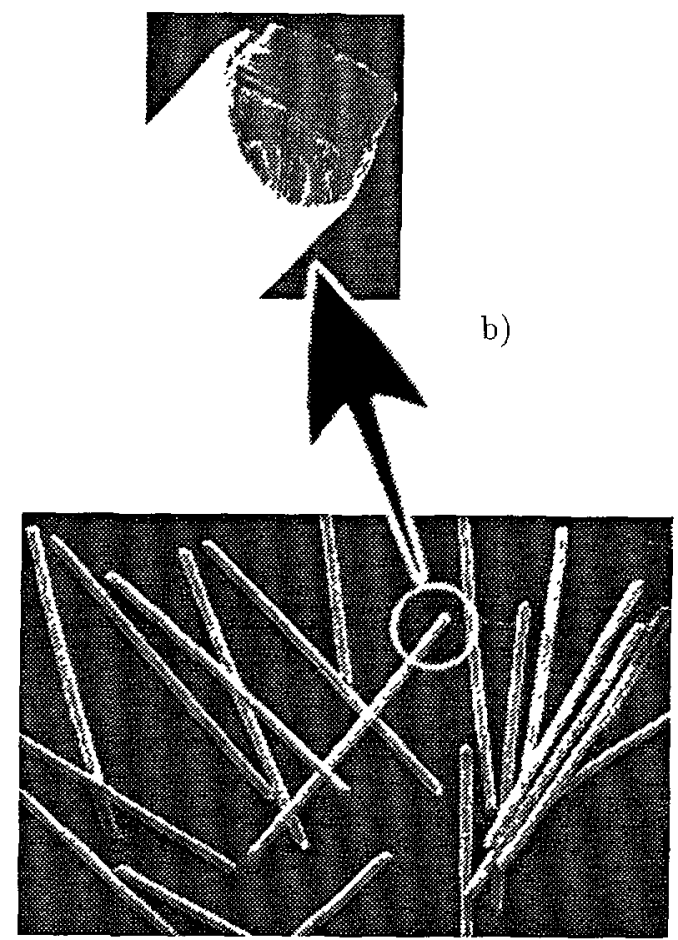

a)

Fig 1 - a) Photo d'un échantıllon de fibres b) Photo de l'extrémité d'une fibre (a) Photo of a sample of the used fibers b) Photo of an extremity of a bronze fiber ]

graduée et un chronomètre sont utilisés pour détermmer le débit volumique $Q$ à la sortie - 1- du réservoir (B) Une fols les valeurs de $Q$ et $\Delta H$ acquises, la hauteur prézométrique est augmentée jusqu'à la valeur désirée, et après stabilisation du système, les valeurs suivantes sont relevées. Cette procédure est répétée sur la gamme des hauteurs piézométrıques accessibles.

Ce dispositıf nous permet de déterminer les perméabilités des différents milieux étudiés et d'analyser les lois d'écoulement suivant les directions parallèle et perpendiculaire à la direction de l'empilement. Pour ce farre, nous avons utılisé deux cellules différentes contenant le milieu poreux

La figure $2 \mathrm{~b}$ représente une vue éclatée de la cellule de mesure spécifique à la détermınatıon des lois d'écoulement et de la perméabilité de l'empilement. Elle est composée de deux réservoirs de tranquillısation de l'écoulement traversant le milıu fibreux. Des filtres constitués de billes de bronze de diamètre $60 \mu \mathrm{m}$ frittées maintiennent les fibres dans la cellule de mesure Pour la réalisation de cette cellule nous avons utılisé du plexiglas pour sa facılité de mise en oeuvre et sa transparence

Les prises de pression sont positionnées à l'intérieur du mılieu fibreux, au voisinage des filtres De ce fait, seule la perte de charge induite par le milieu fibreux est prise en compte

La figure $3 a$ représente la cellule de mesure spécifique à l'étude de l'influence de la direction de l'écoulement sur les lois de perte de charge L'enceinte contenant les fibres a une forme cubique de dimensions $40 \times 40 \times 40 \mathrm{~mm}^{3}$. Deux faces opposées (FS et RS) sont fixes et transparentes 


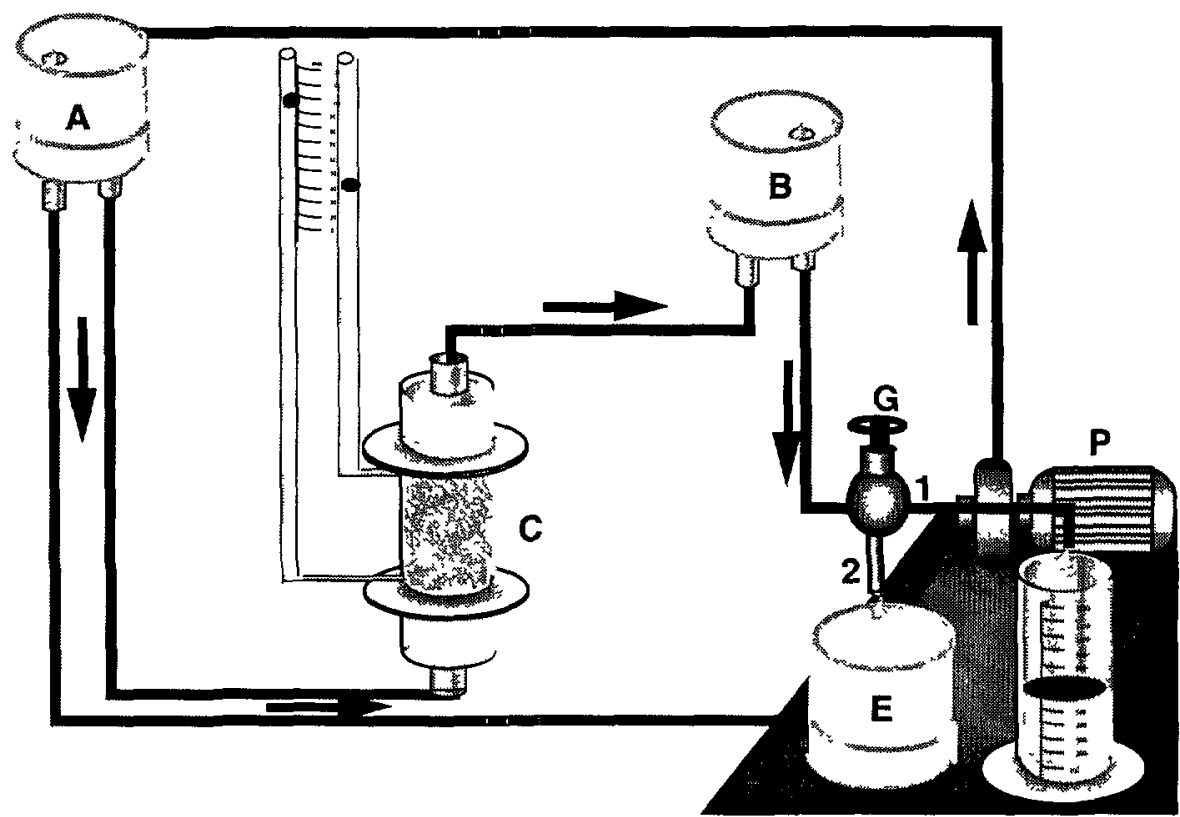

a)

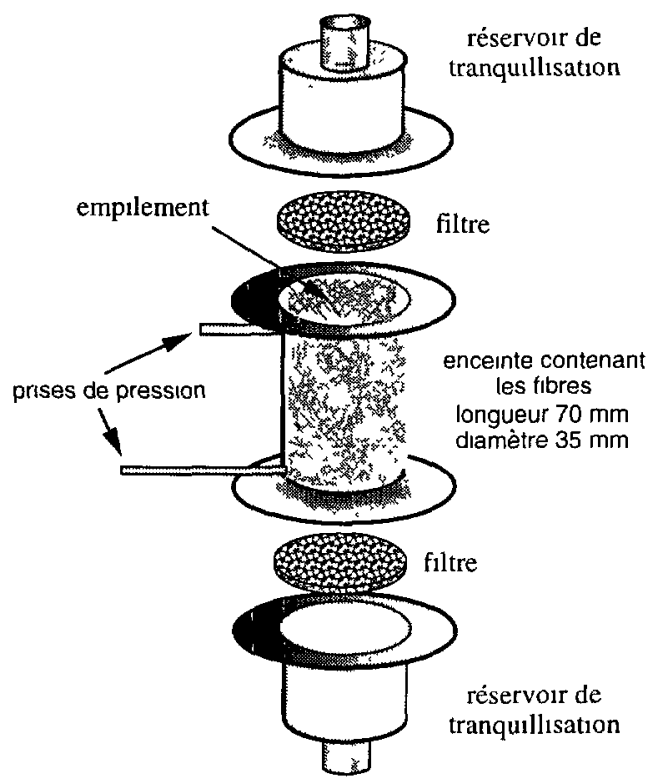

b)

Fıg 2. - a) Schéma du dispositif expérımental pour la détermınatıon de la perte de charge en fonctıon de la vitesse apparente. Cellule contenant les fibres "C" Réservorrs "A, B, E" Pompe péristaltıque "P" Vanne troıs voıes " $G$ " b) Schéma de la cellule utılısée pour la détermınation des perméabılıtés des milieux fibreux

[a) Experimental device for the determination of the pressure drop versus the superficial velocity of a liquid $\mathrm{C}$ Cell containıng the fibers $\mathrm{A}, \mathrm{B}, \mathrm{E}$ Tanks $\mathrm{P}$ Peristaltıc pump $\mathrm{G}$ Multiple-way valve b) Sketch of the cell used for the determination of the permeability of the porous medium ] 


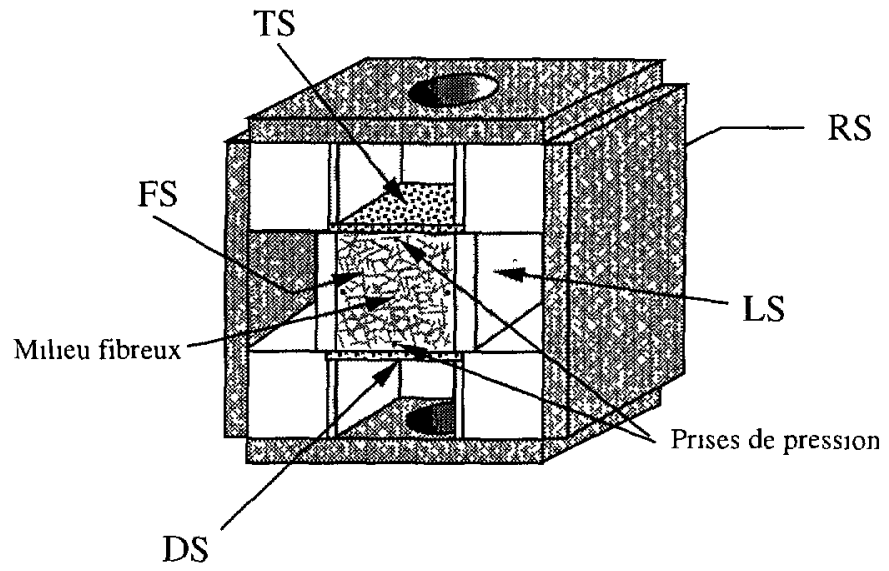

a)

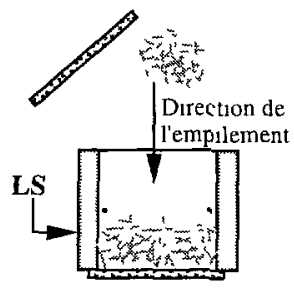

1

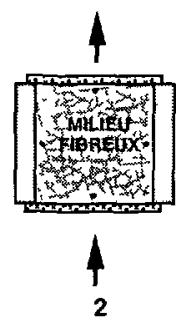

b)

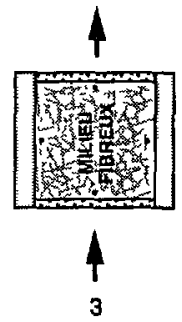

3

Fıg 3 - a) Schéma de la cellule utılısée pour l'étude de l'influence de la directıon de l'écoulement b) Méthode utılısée pour l'étude de l'influence de la direction de l'écoulement 1) remplissage de la cellule ,2) détermination de la loı d'écoulement dans la dırectıon parallèle à celle de l'empilement , 3) détermination de la lor d'écoulement dans la direction perpendıcularre à celle de l'empilement

(a) Sketch of the cell used for analysis of the influence of the flow direction through the porous medium

b) Principle of the method used for analysis of the influence of the flow direction through the porous medium 1) Cell filling 2) Flow direction parallel to the fiber packing direction 3) Flow direction perpendicular to the fiber packing direction ]

Deux autres faces opposés (DS et TS) sont utilısées comme filtres. Les faces latérales (LS) sont des plaques de plexiglas imperméables.

Cette cellule a été conçue de sorte à changer la direction de l'écoulement à travers le milieu sans modifier l'emplement des fibres. Pour cela, après avorr remplı l'enceinte de fibres suivant une direction donnée (Fig $3 b 1$ ), la cellule est insérée dans la boucle expérımentale. Les lois de perte de charge sont ainsi détermmées dans la directıon parallèle à la directıon de l'empilement (Fig. 3b2) La cellule est ensuite tournée de $90^{\circ}$, en permutant les filtres et les faces imperméables (Fig 3b3). 
Les variations de la perte de charge en fonction de la vitesse apparente du fluide sont ainsi détermınées pour les écoulements perpendiculaires à la direction de remplissage de la cellule.

\section{Résultats expérimentaux et discussion}

Il s'agit d'analyser le comportement d'un fluide en écoulement permanent traversant un milieu fibreux. Pour cela, nous déterminons la perméabilité et le paramètre de Kozeny Carman caractérisant l'aptitude du fluide à se mouvoir dans la structure poreuse. Comme les particules sont des cylindres, il est possible d'engendrer des orientatıons préférentielles des fibres lors de l'empilement, pouvant entraîner des modifications des lors d'écoulement suivant les directions étudiées. Une étude préalable a consistée à déterminer ces lois suivant les dırections parallèle et perpendiculaire à la direction de l'empilement

La figure 4 montre la variation de la perte de charge en fonction de la vitesse apparente dans ces deux directions d'écoulement, pour plusieurs rapports d'aspect. Les lois de variation obtenues sont quasiment identiques. Le farble écart constaté aux plus fortes vitesses peut être attribué aux erreurs de mesures. A partir de ces résultats, il semble qu'aucune des directions

$\Delta \mathrm{P} / \mathrm{H}(\mathrm{Pa} / \mathrm{m})\left(* 10^{-4}\right)$

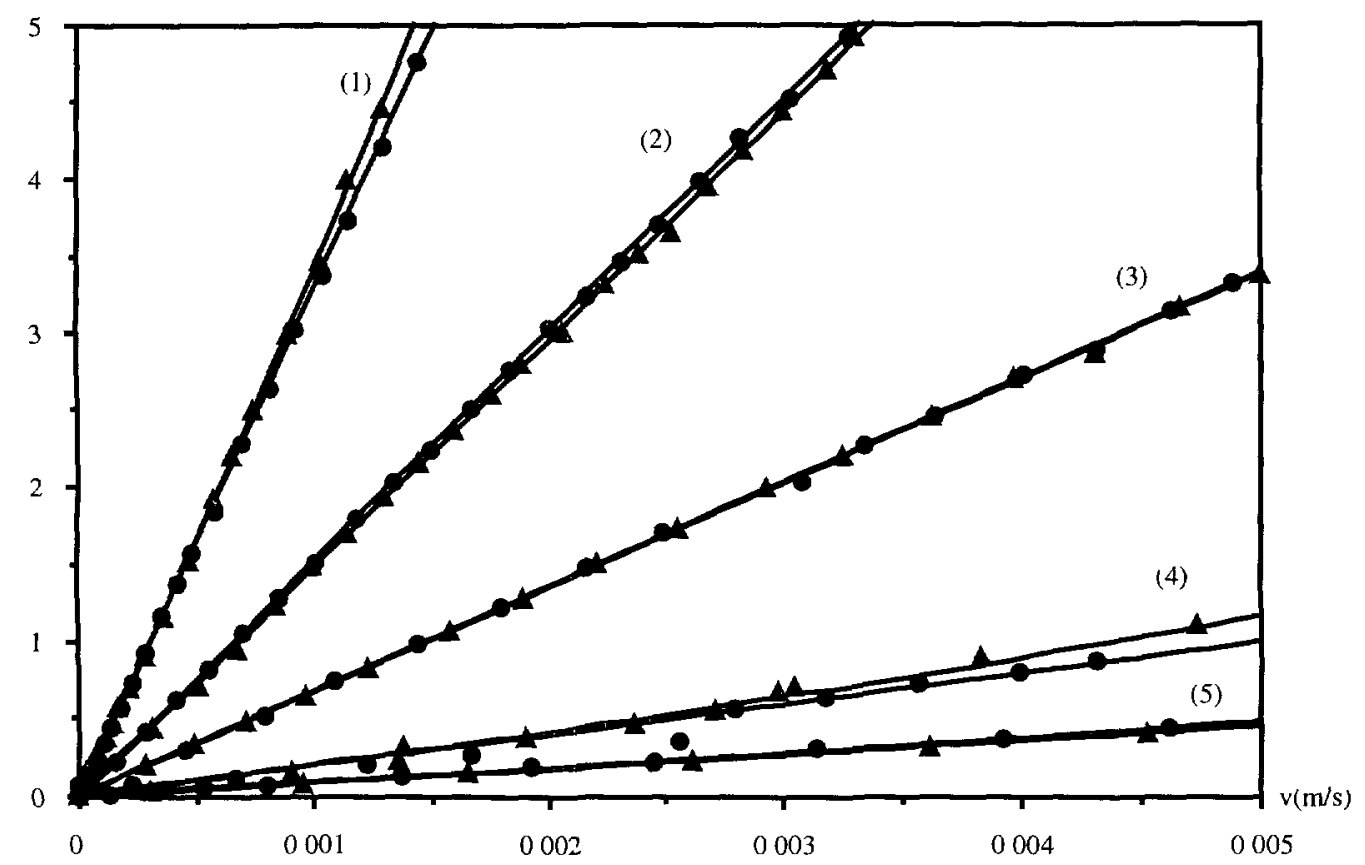

Fig 4 - Variation de la perte de charge en fonction de la vitesse apparente du liquide pour deux directions de l'écoulement, pour différents milieux poreux obtenus (1) $r=4,5$. (2) $r=7,3$, (3) $r=10,7$; (4) $r=18,(5) r=20,2$ ( $\mathbf{\Lambda}$ ) direction de l'écoulement parallèle à la directıon de remplıssage de la cellule (⿶) directıon de l'écoulement perpendicularre à la directıon de remphissage de la cellule [Pressure drop versus the superficial velocity for two mann direction of the liquid flow Aspect ratios of the fibers (1) $r=45$, (2) $r=7.3$, (3) $r=107$, (4) $r=18$, (5) $r=202$ (4) Flow direction parallel to the fiber packing direction ( $\bullet$ Flow direction perpendicular to the fiber packing direction ] 
ne soit privllégiée vis à vis de l'écoulement. Il en ressort que le milieu est macroscopiquement isotrope, au moins pour ce qui concerne la perméabilité.

Nous pouvons donc considérer que la perméabilité est une grandeur scalaire, pouvant être aisément exprimée en fonctıon des grandeurs caractéristiques du milieu poreux (porosité, rapport d'aspect et diamètre des fibres)

D'après Bear [10], il existe deux équations décrivant les lols d'écoulement à travers un milieu poreux :

$$
\begin{aligned}
\text { loi de Darcy [11] } \quad \frac{\Delta P}{H} & =\frac{\mu}{K} U \\
\text { lol de Forchhermer [12] } & \frac{\Delta P}{H}=\alpha U+\beta U^{2}
\end{aligned}
$$

où $U$ est la vitesse apparente et $H$ la longueur du lit de particules.

Dans cette expression, $\alpha U$ et $\beta U^{2}$ représentent respectivement les effets visqueux et inertiels, comme cela a été mentionné par de nombreux auteurs (Ahmed-Sunada [13], Ergun [14], Mac Donald [15], etc...).

Lorsque les effets inertiels $\beta U^{2}$ sont négligeables, l'écoulement est alors Darcéen, et la perméabilité du milieu est donnée par l'expression suivante.

$$
K=\frac{\mu}{\alpha}
$$

A partır de ces considératıons, nous avons approximé les points expérımentaux par un polynôme d'ordre un ou deux survant les cas Nous avons d'abord déterminé les coefficients du polynôme de degré 1 par la méthode des moindres carrés. Avant d'introduire un terme de degré 2 dans le polynôme de régression, le test statistique de Snedecor est appliqué Celui-ci permet de vérifier sı l'amélioration apportée par ce terme est significatıve ou non [16] Dans le cas où l'amélıoration est significatıve, le polynôme de régression retenu est alors de degré 2 avec un niveau de confiance égal à 0,995 . Les coefficients de ce polynôme sont alors redéterminés par la méthode des moindres carrés L'incertitude sur les coefficients des polynômes est définıe par leur écart type [16].

La figure 5 montre deux exemples de variation de perte de charge en fonction de la vitesse apparente Le premier correspond au cas où les effets inertiels sont négligeables devant les effets visqueux (régime Darcéen) Dans le second exemple, les effets inertiels ne peuvent plus être négligés au delà d'une certaine vıtesse Nous avons tracé pour ce dernier cas, sur le même graphe. la partie linéaire déduite du polynôme de régression.

Nous avons porté sur la figure 6 les variations des pertes de charge en fonction de la vitesse apparente du flude (eau) La perte de charge en fonction de la vitesse apparente est d'autant plus grande que le rapport d'aspect des fibres est petit. Pour les fables rapports d'aspect, la lo1 de varıation est quasiment lınéare Les effets inertıels n'ont pu être observés du fait des faibles valeurs des vitesses atteintes. Lorsque le rapport d'aspect augmente, et par conséquent la porosité, les effets inertiels deviennent prépondérants dès les farbles vitesses d'écoulement.

Les résultats obtenus sont regroupés dans le tableau I Dans celui-cr sont portées, pour les différents rapports d'aspect, les porosités et les perméabllités correspondantes

Irmay cité par Bear [10] introduıt une lımite inférieure de validité de la loi de Darcy pour l'écoulement d'un fluide à travers un milieu poreux. D'après cet auteur, ll peut exıster une perte de charge seuil en deçà de laquelle il n'y a pas d'écoulement du fluide. Bear [10] attribue ce seuil à l'existence de mouvements internes (contre courant le long des paross des pores, partıculièrement dans les mılieux constitués de grains fins). 
$\Delta \mathrm{P} / \mathrm{H}(\mathrm{Pa} / \mathrm{m})\left({ }^{*} 10^{-4}\right)$

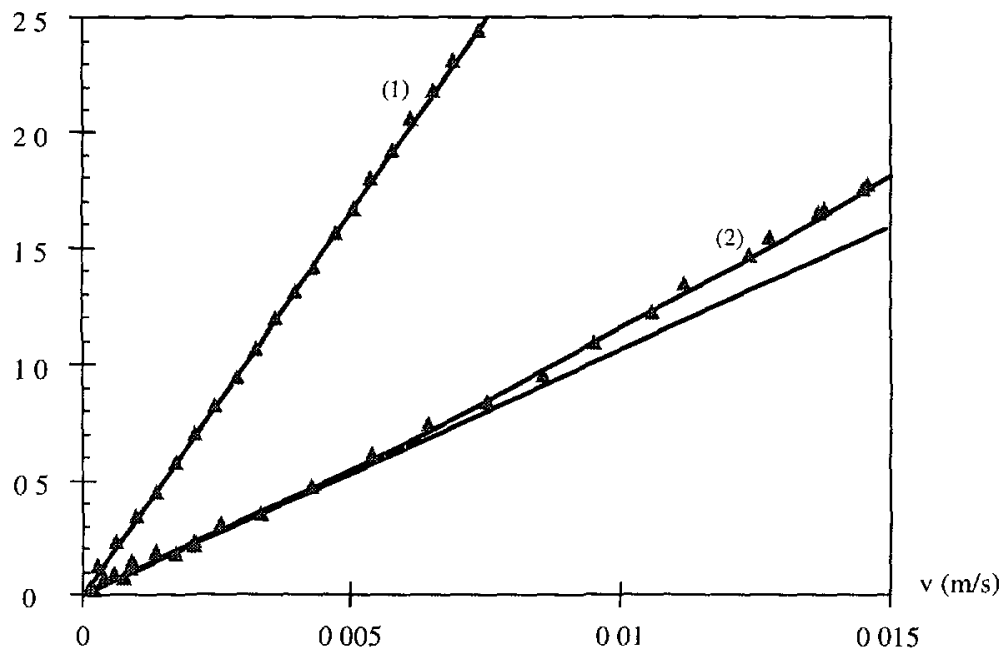

Fıg 5. -- Exemples de varıation de la perte de charge en fonction de la vitesse apparente du fluide (1) effets inertiels néglıgeables (régıme Darcéen), $r=14,7$ (2) régıme non Darcéen, $r=20,2$ Pressure drop versus the superficial velocity of the liquid (1) Inertial effects negligible (Darcean regıme), $r=147$ (2) Non Darcean regıme, $r=202$ ]

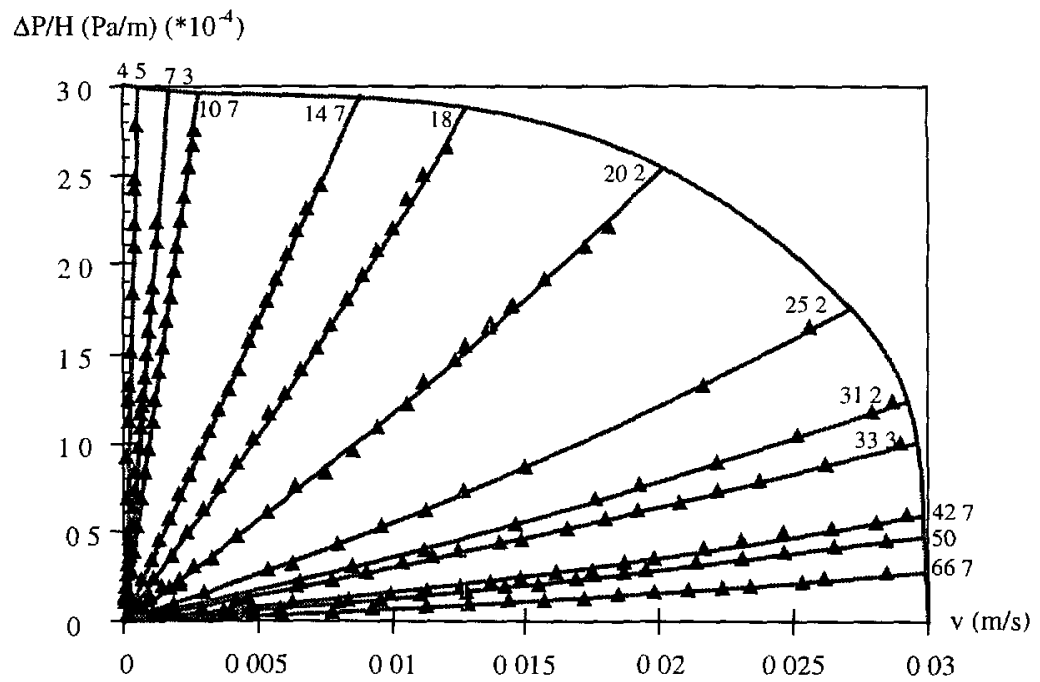

Fig 6 - Variation de la perte de charge en fonction de la vitesse apparente pour l'ensemble des rapports d'aspect étudiés

[Pressure drop versus the superficial velocity of the liquid aspect ratios of the fibers (1) $r=45$, (2) $r=73,(3) r=107$, (4) $r=18,(5) r=20.2$ ] 
Tableau I. - Valeurs expérımentales des paramètres $K$ et $k_{\mathrm{k}}$ pour dufférents rapports d'aspect des fibres et différentes porosités, et erreurs relatıves associées.

[Experimental results of the permeability $K$ and $k_{\mathrm{k}}$ parameter for several aspect ratios of the fibers and the corresponding porosities ]

\begin{tabular}{|c|c|c|c|c|c|}
\hline $\begin{array}{c}\text { rapport d'aspect } \\
r\end{array}$ & $\begin{array}{c}\text { porosité } \\
\varepsilon\end{array}$ & $\begin{array}{c}\text { perméabilıté } \\
K \text { (Darcy) }\end{array}$ & $\begin{array}{c}\text { erreur relative } \\
\Delta K / K(\%)\end{array}$ & $\begin{array}{c}\text { paramètre } \\
k_{\mathrm{k}}\end{array}$ & $\begin{array}{c}\text { erreur relative } \\
\Delta k_{\mathrm{k}} / k_{\mathrm{k}}(\%)\end{array}$ \\
\hline 4,5 & 0,388 & 19 & 1.7 & 9,3 & 19 \\
\hline 7,3 & 0,46 & 60 & 0.5 & 6,8 & 19 \\
\hline 10.7 & 0,523 & 99 & 0,1 & 8,2 & 19 \\
\hline 14,7 & 0,599 & 302 & 0.4 & 5,8 & 21 \\
\hline 18 & 0,642 & 502 & 1 & 5,5 & 23 \\
\hline 20.2 & 0,695 & 938 & 2.6 & 5,1 & 26 \\
\hline 25,2 & 0,736 & 1807 & 1,2 & 4,3 & 27 \\
\hline 31.2 & 0,77 & 2716 & 2,2 & 4,3 & 30 \\
\hline 33,3 & 0.801 & 3654 & 1,8 & 4.9 & 32 \\
\hline 42,7 & 0,844 & 8134 & 4,2 & 4.2 & 40 \\
\hline 50 & 0,851 & 9156 & 3 & 4,2 & 40 \\
\hline 66,7 & 0,891 & 19728 & 6,2 & 4,2 & 52 \\
\hline
\end{tabular}

Dans le but de vérifier l'existence de tels phénomènes, nous avons étudié les varıations de la perte de charge seuil déduite du polynôme de régression. Nous avons représenté, sur la figure 7 , les valeurs de ces ordonnées à l'origine en fonction de la porosité du milieu. Nous remarquons une dispersion des points au voisinage de zéro La valeur moyenne est de $40 \mathrm{~Pa} / \mathrm{m}$. Cette valeur étant du même ordre de grandeur que l'incertitude sur les mesures de perte de charge, aucune conclusion ne peut être apportée concernant ce point. Nous conservons donc les lois de régression s'exprimant sous la forme des lo1s de Darcy ou Forchheimer.

Les perméabilités dédultes des résultats expérımentaux varient en fonction de la porosité $\epsilon(L / d)$ (Fig 8). Cette variation est d'autant plus prononcée que la porosité du milieu est importante. La perméablité varie de 20 à 20000 Darcy lorsque la porosité varie de 35 à $90 \%$ La forte variation de cette grandeur avec la porosité rend difficile une analyse fine de nos résultats expérimentaux. Nous avons, donc, chossi d'étudier le paramètre de Kozeny Carman " $k_{\mathrm{k}}$ " qui varie dans une gamme beaucoup plus farble.

De nombreux modèles visant à prédire la perméabilité d'un milieu poreux en fonction des caractéristıques géométriques ont été proposés dans la littérature ([17,18], etc .. ). Ces modèles sont basés sur des géométrıes sımplifiées. Certains considèrent des capillaires de sections uniformes ou différentes D'autres supposent des écoulements à travers des cylındres rangés en réseaux séries, parallèles ou une combinaison de ces arrangements. A notre connalssance, seuls les modèles de Kyan [9] et de Jackson et James [3] vısent à prédıre la perméabılité d'un empllement aléatorre de fibres. D'une manière générale, les auteurs relient leurs résultats, tant numérıques qu'expérimentaux, à la porosité du milieu et à la surface spécifique des particules en utilisant la relation de Kozeny Carman La constante $k_{\mathrm{k}}$ qui apparaît dans cette expressıon est alors détermınée pour les différents milieux étudiés. Dans le cadre de cette étude, 
ordonnẻe à l'orıgine $(\mathrm{Pa} / \mathrm{m})$

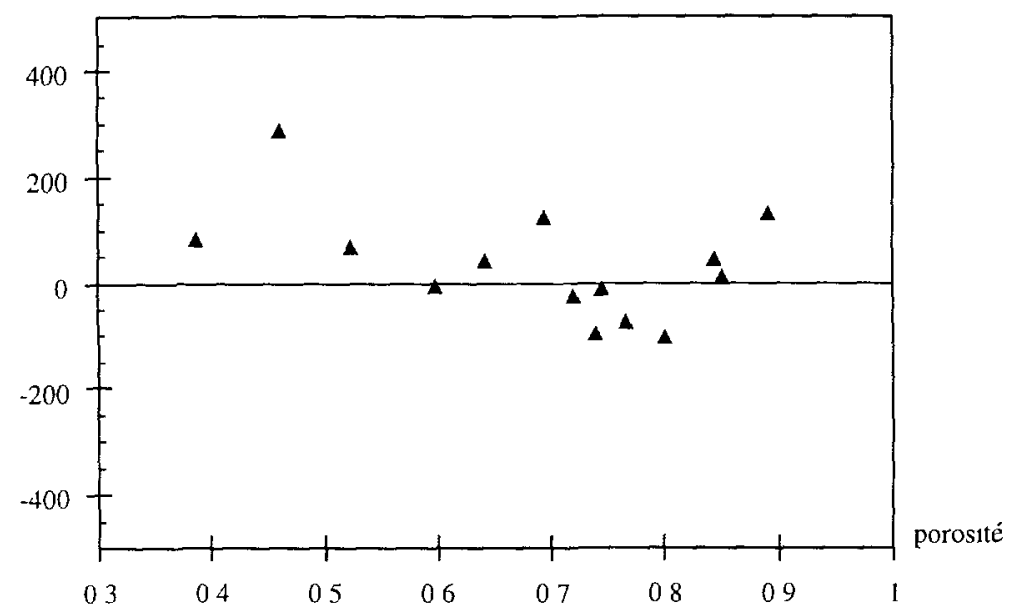

Fig 7 - Varıation de l'ordonnée à l'orıgıne des loıs de régression en fonction de la porosité $\epsilon$ du milieu fibreux

[Variation of the ordinate at $V=0$ of the fitted curve versus the porosity of the fibrous medium ]

nous avons adopté la même démarche dans le cas de l'emplement aléatoire de fibres monodisperses. Nous avons ensuite étudié les variations du paramètre de Kozeny Carman $k_{\mathrm{k}}$, déduit des perméabılités expérımentales et de la relatıon survante

$$
K=\frac{\epsilon^{3}}{k_{\mathrm{k}}(1-\epsilon)^{2} a_{\mathrm{p}}^{2}}
$$

où $k_{\mathrm{k}}$ est la constante de Kozeny Carman, $a_{\mathrm{p}}$ la surface spécifique des partıcules et $\epsilon$ la porosité du milieu poreux. Cette expression a été déduıte par Kozeny Carman [18], à partir d'un modèle assımilant l'espace interstıtiel du milieu poreux à un ensemble de capillaires droits, cylindrıques de même diamètre et de longueur supérieure à celle de l'échantillon.

Pour les fibres utilisées dans cette étude, la surface spécifique est donnée par la relation sulvante .

$$
a_{\mathrm{p}}=\frac{2(2 r+1)}{r d}
$$

où $r$ est le rapport d'aspect de la fibre $(L / d)$

La perméabilité $K$ du milieu poreux est alors donnée par.

$$
K=\frac{\epsilon^{3} r^{2} d^{2}}{4 k_{\mathrm{k}}(1-\epsilon)^{2}(2 r+1)^{2}}
$$

La relation (11) nous permet donc de déterminer, à partir des résultats expérimentaux obtenus, les variations du paramètre $k_{\mathrm{k}}$ en fonction de la porosité $\epsilon(L / d)$. Ces valeurs sont regroupées dans le tableau. Les erreurs indiquées sont estimées par la relation

$$
\frac{\Delta k_{\mathrm{k}}}{k_{\mathrm{k}}}=3 \frac{\Delta \epsilon}{\epsilon}+2 \frac{\Delta r}{r}+\frac{\Delta K}{K}+2 \frac{\Delta \epsilon}{(1-\epsilon)}+4 \frac{\Delta r}{(2 r+1)}
$$


Permeabılıté K (Darcy)

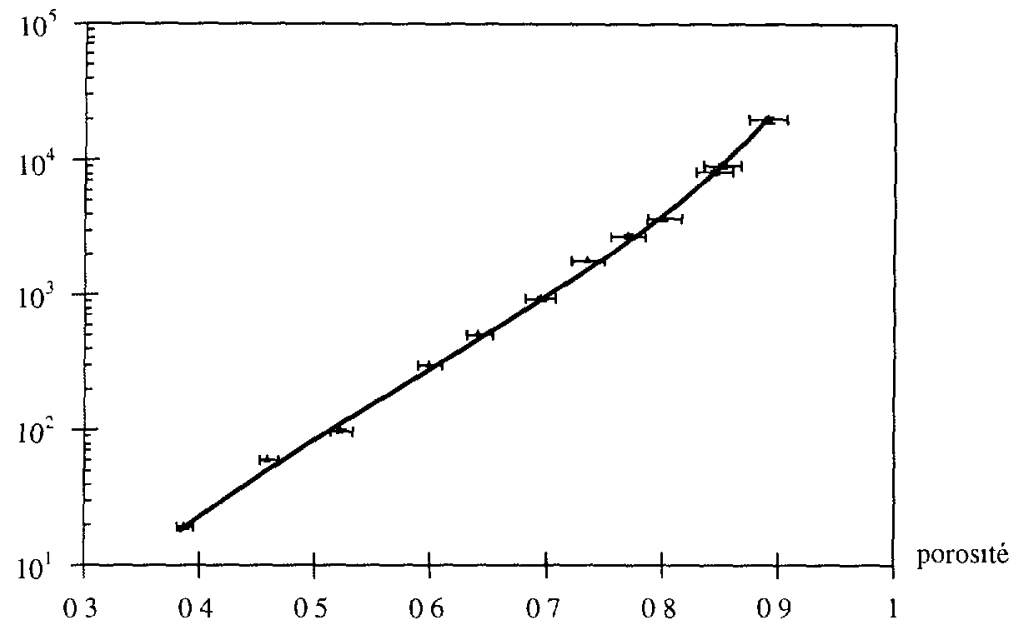

Fig 8 - Varıation de la perméabılıté en fonctıon de la porosité $\epsilon$ du milıeu fibreux Fibres (diamètre $=150 \mu \mathrm{m}$ et $4,5 \leq r \leq 66,7$ )

[Fibrous medium permeability versus the porosity (Fibers diameter $150 \mu \mathrm{m}$, aspect ratio $45<r<$ $66.7)]$

L'erreur sur la porosité est essentiellement liée à l'erreur commise sur la hauteur du lit, estimée à $\pm 0,5 \mathrm{~mm}$ L'incertıtude sur la longueur des fibres est 1ci égale à 2,5\%, tenant compte des erreurs dues aux extrémités des fibres lors de la découpe, et à la lecture des mesures de ces longueurs.

Sur les figures 9 et 10 , sont représentées respectivement les valeurs de $k_{\mathrm{k}}$ pour les différentes valeurs de porosité et du rapport d'aspect. Nous avons associé à chaque point de mesure l'incertitude correspondante. Nous remarquons que les évolutions de ce paramètre en fonction de $r$ et $\epsilon$ sont simılaires Elles sont contınûment décroissantes avec une tendance asymptotique. $\mathrm{Au}$ vu de ces varıations, nous avons approximé ces points par des fonctions hyperboliques simples d'expressions.

$$
\begin{gathered}
k_{\mathrm{k}}=\frac{3,6}{\epsilon} \\
k_{\mathrm{k}}=3,6+\frac{30}{r}
\end{gathered}
$$

Ces lois corrèlent relatıvement bien l'ensemble des points expérımentaux La valeur asymptotique de $k_{\mathrm{k}}$ pour $r$ tendant vers l'infinı $(\epsilon=1)$, égale à 3,6 , se rapproche de celle donnée par la loi d'Ergun modifiée égale à 4,17 .

Cette loı de variation de $k_{\mathrm{k}}$ est à rapprocher de celle de la contrıbution des surfaces de base à la surface totale de la fibre .

$$
\gamma=\frac{\text { surfaces de base }}{\text { surface totale }}=\frac{1}{2 r+1}
$$

Cette loi de variation est de type hyperbolique, comme pour $k_{\mathrm{k}}$ La contribution des surfaces de base devient inférieure à $2 \%$ pour $r$ supérieur à 25 On peut constater sur la figure 11 que la valeur de $k_{\mathrm{k}}$ reste sensiblement constante au delà de cette valeur de $r$. Il semble donc que 


$$
\mathbf{k}_{\mathrm{h}}
$$

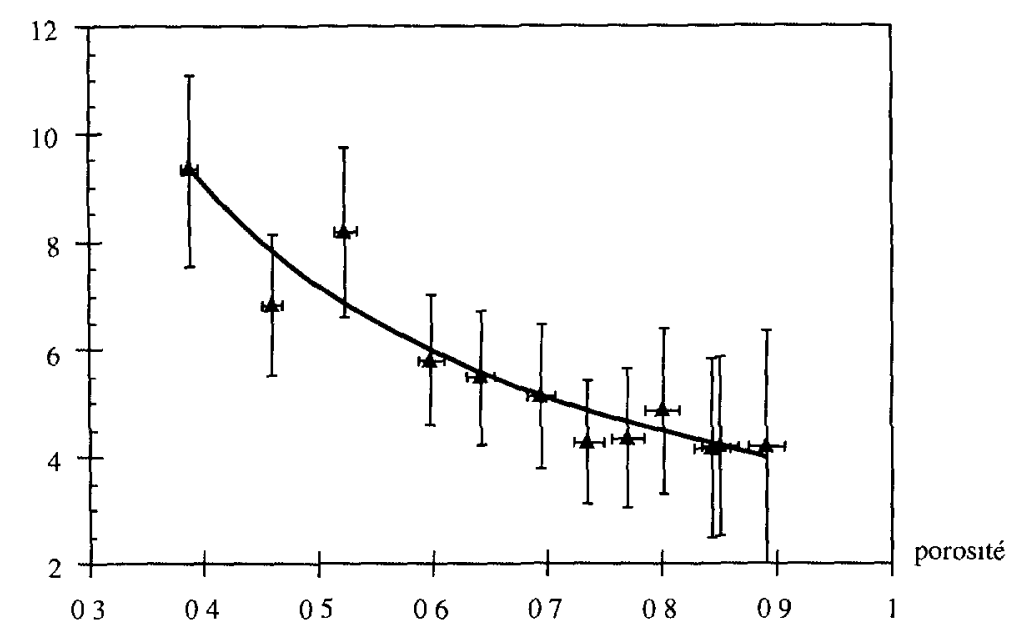

Fıg 9. - Variation du paramètre $k_{\mathrm{k}}$ en fonction de la porosité du milıeu fibreux. Fibres (diamètre $=150 \mu \mathrm{m}$ et $4,5 \leq r \leq 66,7$ ).

[Varıation of the $k_{\mathrm{k}}$ parameter versus the fibrous medıum porosity (Fibers diameter $150 \mu \mathrm{m}$, aspect ratio $4.5<r<667)$ ]

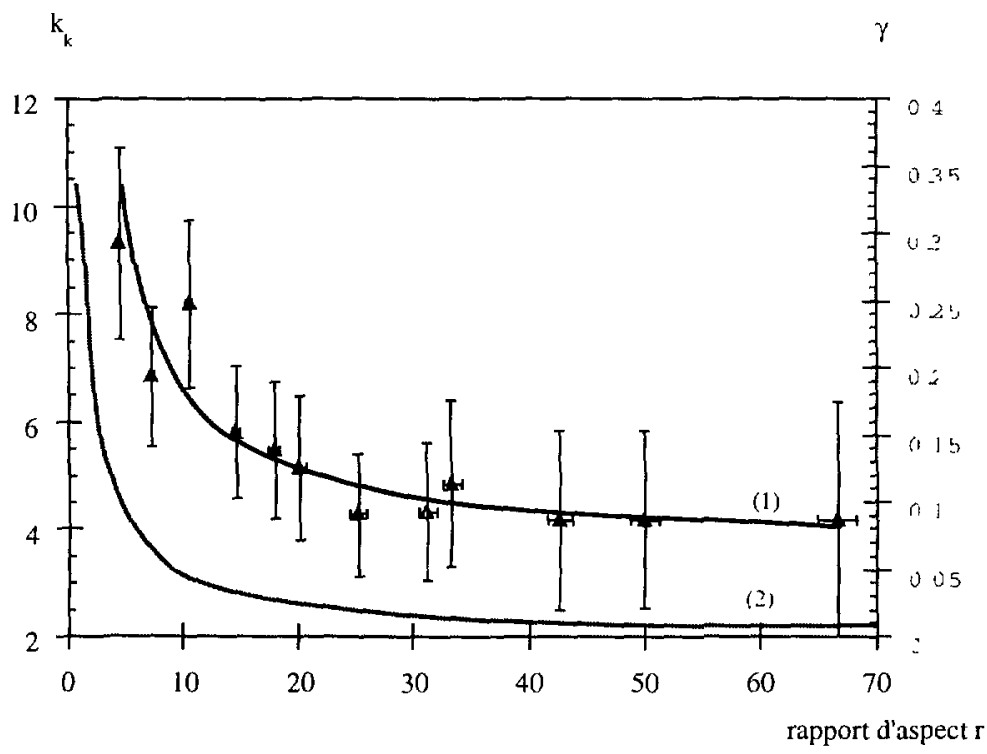

Fig 10. - Varıation du paramètre $k_{\mathrm{k}}$ (1) et de la contribution $\gamma$ des surfaces de base des fibres (relation (15)) (2) en fonction du rapport d'aspect

[Variation of the $k_{\mathrm{k}}$ parameter (curve 1) and the lateral areas (Eq (15)) (curve 2) of the fibers versus the aspect ratio of the fiber ] 
Perméabılité K (Darcy)

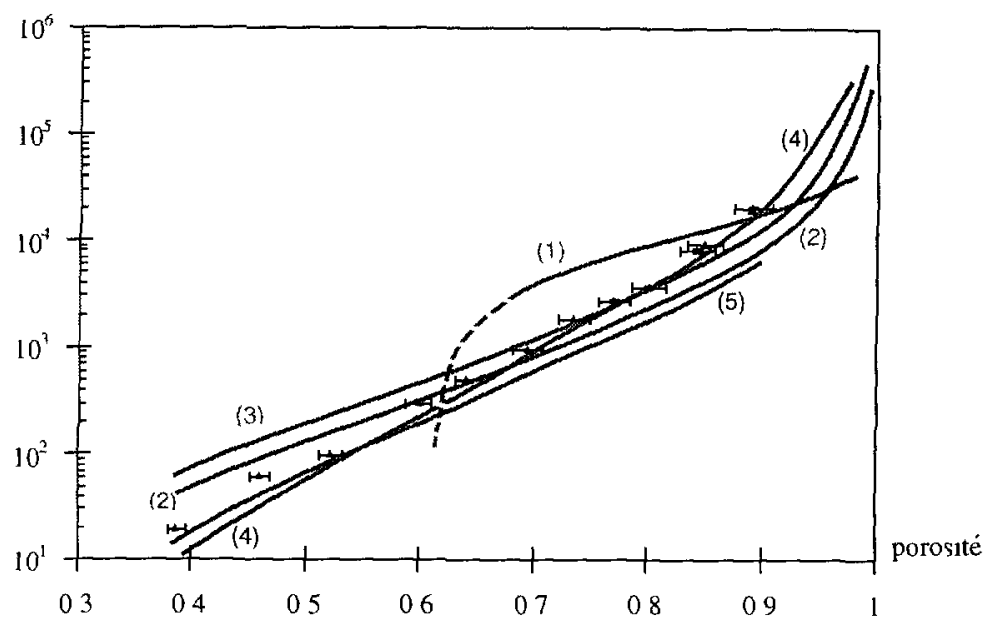

Fıg 11. -- Vauation de la perméabilité en fonction de la porosité $\epsilon$ du milieu fibreux (ム) nos résultats expérımentaux, (1) modèle de Jackson et James [3] , (2) modèle de Happel et Brenner (perpendıculaıre) [7], (3) modèle de Happel et Brenner (parallèle) [7], (4) modèle de Kyyan [9] , (5) modèle de Sahraoui et Kavıany [20]

[Permeability variation versus the porosity of the porous medium (4) Experimental results of the present work, (1) Jackson and James model [3], (2) Happel and Brenner model (flow perpendicular to the cylinders) [7], (3) Happel and Brenner model (flow parallel to the cylinders) [7], (4) Kyan model [9], (5) Sahraous and Kaviany model [20].]

les surfaces de base jouent un rôle non néglıgeable dans les varıations de $k_{\mathrm{k}}$ pour les farbles rapport d'aspect $(r<25)$

A partır des relatıons (13) et (14), et de la relatıon (11) déduite du modèle de Kozeny Carman, nous proposons la relation semi empirique suivante relıant la perméabilité du milıeu étudié à sa porosité

$$
K=\frac{62,5 \epsilon^{6} d^{2}}{(1-\epsilon)^{2}(3,6+56,4 \epsilon)^{2}}
$$

Cette relation décrit correctement les variations de la perméabilité expérimentale en fonction de la porosité $\epsilon$ (Fig. 8).

\section{Discussion et confrontation avec des modèles}

A notre connarssance, il existe un nombre limité de travaux traitant de la perméabilité de milieux poreux fibreux Par ailleurs, la dénomınation de milieux fibreux englobe une multitude de milieux poreux de textures différentes (filtre, lane de verre, tissus, etc ...). Concernant les milıeux constıtués d'emplements aléatoires de fibres rigıdes monodisperses, il n'existe, à notre connaissance, pas de travaux publiés à ce jour.

Dans ce paragraphe, nous tentons de comparer les résultats obtenus à ceux proposés par divers auteurs, disponibles dans la littérature pour des milieux fibreux définis au sens large Jackson et James [3] proposent un modèle donnant la perméabilité en fonction de la porosité Ce modèle permet d'obtenır une expressıon de la perméabilité pour des empilements aléatoures 
de fibres en fonction de la porosité (relation (1)). Ces auteurs précisent que cette loi est valable seulement pour des porosités élevées $(\epsilon>0,7)$.

Kaviany [19] déduit le paramètre de Kozeny Carman à partır des travaux d'Happel et Brenner [7] dans le cas d'un fluide en écoulement parallèle ou perpendiculaire à l'axe d'un cylindre. Il exprime $k_{\mathrm{k}}$ en fonction de la porosité (relations (2) et (3)) Par combinaison des relations (2) et (9), et (3) et (9), respectivement pour un écoulement parallèle et perpendiculaire, nous déduisons les évolutions de la perméabilité en fonction de la porosité.

Nous avons porté sur la figure 11 les lois déduites de ces modèles, ainsi que nos résultats expérimentaux.

Les perméabilités données par le modèle de Jackson et James [3] sont supérieures à nos valeurs pour des porosités comprises entre 0,7 et 0,85 La loi de variation proposée par ces auteurs diffère de celle déduite de nos expériences dans toute la gamme de porosité explorée.

Les résultats déduits des modèles d'Happel et Brenner [7] donnent des lois de comportement analogues à celle de nos expériences avec des écarts sensiblement importants suivant les valeurs de la porosité Pour des porosités inférieures à 0,6 , ces modèles surestiment les valeurs de la perméabilité. Pour des porosités supérieures à 0,7 , les perméabilités calculées sont inférieures.

Par combinaison des relations (4), (5) et (9), nous dédulsons l'évolution de la perméabilité, déduite du modèle de Kyan et al [9], en fonction de la porosité

En outre, des solutions numériques de l'équation de Navier-Stokes ont été déterminées par Sahraoui et Kaviany [20] pour un écoulement à travers un réseau de cylindres. Ils déduisent, des résultats obtenus, la lor de varıation de la perméabilıté en fonction de la porosité .

$$
h=0,0606 \frac{\pi}{4} \frac{\epsilon^{5,1}}{1-\epsilon} d^{2} \quad 0,4 \leq \epsilon \leq 0,8
$$

Les perméabilités déduites de ces modèles sont comparées à celles déterminées à partir de nos expérıences (Fig. 11).

Une bonne concordance est observée entre nos résultats est ceux de Kyan et al. [9] pour des porosités comprises entre 0,7 et 0,9 , où les courbes se tangentent. Pour des porosités inférieures, un écart est observé. Par contre, les résultats numériques de Sahraoul et Kaviany [20] sont systématıquement inférieurs avec des écarts d'autant plus importants que la porosité est élevée.

Comme nous l'avons vu précédemment, la perméabilité varie de plusieurs ordres de grandeur. Celle-cı dépend de plusieurs paramètres (relatıon (9)) et en particulier du paramètre $k_{\mathrm{k}}$ caractérisant la trajectorre du fluide à travers le milıeu poreux. Dans le but de donner une interprétation et d'effectuer une comparaison plus fine des résultats, nous avons étudié les varıations du paramètre $k_{\mathrm{k}}$ en fonction de la porosité du milieu.

Nous avons comparé les paramètres $k_{\mathrm{k}}$ déduits des divers modèles et de nos expériences (Fig. 12). Nous remarquons qu il existe deux types de comportement suivant les modèles .

- une tendance contınúment crolssante avec des variatıons importantes lorsque la porosité est supérieure à 0,85 ([7] d’après [19] et [3]). Nos expériences donnent une tendance inverse,

- une tendance parabolique présentant un minimum. Sahraoui et Kaviany [20] obtiennent un minimum pour une porosité de $0 . \bar{\tau}$, tandis que pour Kyan [9], ce minimum se situe à 0.85 Pour les valeurs de porosité inférieures au minimum, la tendance décroissante est aussi observée dans nos expériences Dans tous les cas nous n'observons pas de croissance dans la gamme des porosités explorées

Les lois de varıation de $k_{\mathrm{k}}$ en fonctıon de la porosıté sont difficıles à interpréter Notons cependant que les courbes donnant une variation de $k_{\mathrm{k}}$ croissante avec la porosité sont obtenues 


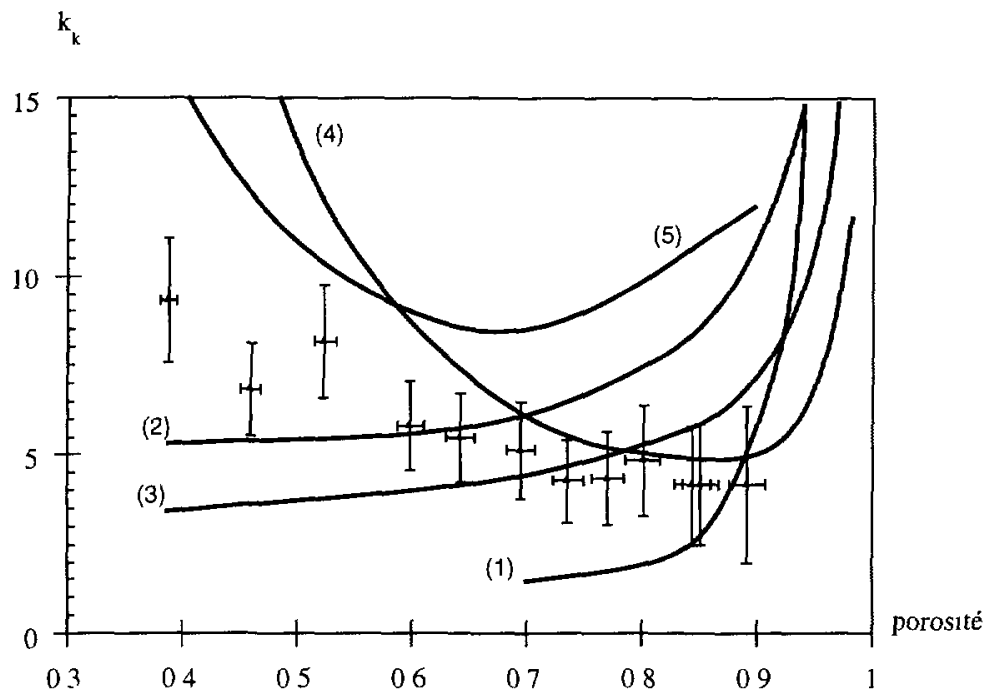

Fig 12 - Variation du paramètre $k_{\mathrm{h}}$ en fonction de la porosité $\epsilon$ du milieu fibreux. ( $\mathbf{\Delta}$ ) nos résultats expérimentaux , (1) modèle de Jackson et James [3] , (2) modèle de Happel et Brenner (perpendiculaire) [7] , (3) modèle de Happel et Brenner (parallèle) [7], (4) modèle de Kyan [9] , (5) modèle de Sahraou et Kaviany [20]

$\left\{k_{\mathrm{k}}\right.$ parameter variation versus the porosity of the porous medium (4) Experimental results of the present work: (1) Jackson and James model [3], (2) Happel and Brenner model (flow perpendicular to the cylinders) [7], (3) Happel and Brenner model (flow parallel to the cylinders) [7], (4) Kyan model [9], (5) Sahraouı and Kaviany model [20]]

par addition des pertes de charge occasionnées par chaque particule Les courbes présentant un minimum sont, quant à elles, obtenues à partir d'évaluation des pertes de charge directement dans un réseau de cylindres ordonnés.

La décroissance du paramètre $k_{\mathrm{k}}$ en fonction de la porosité, obtenue pour un empilement aléatorre de fibres monodisperses traversé par un liquide, peut être interprétée par le fait que les trajectorres du fluide ont tendance à devenir rectilignes au fur et à mesure que la porosité augmente. Les valeurs élevées de $k_{\mathrm{k}}$, lorsque les surfaces de base des fibres sont importantes, semblent indiquer que leur contribution, dans la trajectoire du fluide, n'est pas négligeable.

Ces différentes comparaisons montrent que les modèles basés sur des fibres disposées en réseaux simples et bien ordonnés ne prédısent pas correctement les perméabilités des empilements aléatoires de fibres rigides monodısperses

En effet, il est difficile d'admettre une augmentation du paramètre $k_{\mathrm{k}}$ avec la porosité (dans la gamme de fortes porosités) étant donné que ce paramètre est l'image de la tortuosité

\section{Conclusion}

Dans cet article, nous avons étudié les écoulements dans des milıeux poreux constitués d'empilements aléatoires de fibres rigides monodisperses. Une étude systématique en fonction de la porosité $\epsilon(L / d)$ a été réalisée.

L'étude de l'mfluence de la direction de l'écoulement nous a permis de montrer que les empilements aléatoires de fibres monodısperses sont globalement ısotropes vis à vis de l'écoulement. 
A partir des lois d'écoulements expérımentales et de la lor de Darcy, nous avons déterminé les variations des perméabilités en fonctıon de la porosité du milieu.

En généralısant l'équation de Kozeny Carman à des empilements de partıcules cylindriques, nous avons déterminé l'évolution du paramètre $k_{\mathrm{k}}$ en fonction de la porosité $\epsilon(L / d)$. L'évolution de ce paramètre $k_{\mathrm{k}}(\epsilon)$ est continûment décrolssante, avec une tendance asymptotique vers une valeur voisine de 3,6 lorsque la porosité tend vers l'unité.

La comparaison de nos résultats expérimentaux avec des modèles considérant des cylindres disposés en réseaux simples, n'est pas satisfaisante, quelle que soit l'approche proposée

Une modélisation, prenant en compte l'aspect aléatolre de l'empllement des fibres, semble donc nécessaire à développer pour prédire les écoulements dans ce type de milieux.

\section{Remerciements}

Les auteurs remercient $\mathrm{R}$ Blanc pour les nombreuses discussions fructueuses et $\mathrm{R}$. Faure pour son aide technique anns que l'ECOTECH-CNRS pour son ande financière

\section{Bibliographie}

[1] Rahlı O, Tadrıst L, Santını R and Pantalonı J, "Fluıd Flow and Heat Transfer Analysis in F1brous Porous Media", 3rd World Conference on Experimental Flurd Mechanics and Heat Transfer, Honolulu-USA (1993)

[2] Milewskı J V, Adv Ceramıc Mater, 1 (1986) 36

[3] Jackson G. W and James D. F, Can J Chem Eng 64 (1986).

[4] Carman P C, Soc Chem Indust (Trans and Commun) 57 (1938) 225-234

[5] Wiggins E J., Campbell W. B and Maass O, Can J Res B 17 (1939) 318-324

[6] Drummond J E and Tahır M I, Int J. Multaphase Flow 10 (1984) 515-540

[7] Happel $\mathbf{J}$ and Brenner H, Low Reynolds Number Hydrodynamics (Martunus Nijhoff Publishers, 1986)

[8] Porous Media Fluid Transport and Pore Structure (Academıc Press, 1979)

[9] Kyan C P, Wasan D T and Kintner R C, Ind Eng Chem Fundam 9 (1970) 596

[10] Bear J , Dynamics of Fluıds in Porous Media (Dover Publications, 1988)

[11] Darcy H, Les Fontaines Publiques de la Ville de Dijon (Dalmont, Paris, 1856)

[12] Forchheımer P H , Z Ver Disch Ing 49 (1901) 1736-1749, 50 (1901) 1781-1788

[13] Ahmed N and Sunada D. K, J Hydraul Div Proc ASCE 95 (1969) 1847

[14] Ergun S, Chem Eng Prog 48 (1952) 89-94

[15] Mac Donald I F, El-Sayed M S., Mow K. and Dullien F A. L, Ind Chem Fundam 18 (1979)

[16] Neully $M$ et CETAMA, Modélısation et Estimatıon des Erıeurs de Mesure (Technique et Documentation, Lavoisier, 1993)

[17] Dullien F A L, Porous Media Fluid Transport and Pore Structure (Academic Press, second edition, 1992)

[18] Kozeny J, Sitzungsber Akad Wiss. Wien 136 (1927) 271-306

[19] Kaviany M, Mechan Eng Serzes (Springer-Verlag, 1991)

[20] Sahraouı M. and Kavıany M, Int J Heat Mass Transfer 35 (1992) 927-943 